Open J. Math. Anal., Vol. 2(2018), No. 1, pp. 19 - 32

Website: https://pisrt.org/psr-press/journals/oma/

ISSN: 2616-8111 (Online) 2616-8103 (Print)

http://dx.doi.org/10.30538/psrp-oma2018.0009

\title{
SOME NEW HERMITE-HADAMARD-FEJÉR TYPE INEQUALITIES FOR HARMONICALLY CONVEX FUNCTIONS
}

\author{
SERCAN TURHAN ${ }^{1}$, IMDAT İSCAN
}

\begin{abstract}
In this paper, we gave the new general identity for differentiable function. As a result of this identity some new and general fractional integral inequalities for differentiable harmonically convex functions are obtained.
\end{abstract}

AMS Mathematics Subject Classification: 26D15, 26A51, 26D10, $26 \mathrm{~A} 15$. Key words and phrases: harmonically-convex; hermite-hadamard-Fejer type inequalities; fractional integral.

\section{Introduction}

The classical or the usual convexity is defined as follows: A function $f: \emptyset \neq I \subseteq$ $\mathbb{R} \longrightarrow \mathbb{R}$, is said to be convex on $I$ if inequality

$$
f(t x+(1-t) y) \leq t f(x)+(1-t) f(y)
$$

holds for all $x, y \in I$ and $t \in[0,1]$. A number of papers have been written on inequalities using the classical convexity and one of the most fascinating inequalities in mathematical analysis is stated as follows

$$
f\left(\frac{a+b}{2}\right) \leq \frac{1}{b-a} \int_{a}^{b} f(x) d x \leq \frac{f(a)+f(b)}{2},
$$

where $f: I \subseteq \mathbb{R} \longrightarrow \mathbb{R}$ be a convex mapping and $a, b \in I$ with $a \leq b$. Both the inequalities hold in reversed direction if $f$ is concave. The inequalities stated in (1) are known as Hermite-Hadamard inequalities. For more results on (1) which

Received 09-01-2018. Revised 31-01-2018.

1 Corresponding Author

(C) 2018 Sercan Turhan, İmdat İşcan. This is an open access article distributed under the Creative Commons Attribution License, which permits unrestricted use, distribution, and reproduction in any medium, provided the original work is properly cited. 
provide new proof, significantly extensions, generalizations, refinements, counterparts, new Hermite-Hadamard-type inequalities and numerous applications, we refer the interested reader [1, 2, 3, 4] and the references therein. Many researchers extend their studies to Hermite-Hadamard type inequalities involving fractional integrals not limited to integer integrals. The usual notion of convex function have been generalized in diverse manners. Some of them is the so called harmonically $s$-convex function and harmonically convex function is stated in the definition below.

Definition 1.1. [5] A function $f: I \subset(0, \infty) \longrightarrow \mathbb{R}$ is said to be harmonically$s$-convex function if

$$
f\left(\frac{x y}{t x+(1-t) y}\right) \leq t^{s} f(y)+(1-t)^{s} f(x)
$$

holds for all $x, y \in I$ and $t \in[0,1]$, and for some fixed $s \in(0,1]$.

In [6], İşcan gave definition of harmonically convex functions and established following Hermite-Hadamard type inequality for harmonically convex functions as follows:.

Definition 1.2. 6] A function $f: I \subseteq \mathbb{R} \backslash\{0\} \longrightarrow \mathbb{R}$ is said to be harmonicallyconvex function on $I$ if

$$
f\left(\frac{x y}{t x+(1-t y)}\right) \leq t f(y)+(1-t) f(x)
$$

holds for all $x, y \in I$ and $t \in[0,1]$. If the inequality is reversed, then $f$ is said to be harmonically concave.

Proposition 1.3. [6] Let $I \subset \mathbb{R} \backslash\{0\}$ be a real interval and $f: I \rightarrow \mathbb{R}$ is function, then:

if $I \subset(0, \infty)$ and $f$ is convex and nondecreasing function then $f$ is harmonically convex.

if $I \subset(0, \infty)$ and $f$ is harmonically convex and nonincreasing function then $f$ is convex.

if $I \subset(-\infty, 0)$ and $f$ is harmonically convex and nondecreasing function then $f$ is convex.

if $I \subset(-\infty, 0)$ and $f$ is convex and nonincreasing function then $f$ is harmonically convex.

For the properties of harmonically-convex functions and harmonically-s-convex function, we refer the reader to [5, 6, 7, 8, 9, 10, 11, 12, 13, 14, and the reference there in. Most recently, a number of findings have been seen on Hermite-Hadamard type integral inequalities for harmonically-convex and for harmonically-s-convex functions.

Definition 1.4. A function $g:[a, b] \subseteq \mathbb{R} \backslash\{0\} \longrightarrow \mathbb{R}$ is said to be harmonically symmetric with respect to $2 a b / a+b$ if

$$
g(x)=g\left(\frac{1}{\frac{1}{a}+\frac{1}{b}-\frac{1}{x}}\right)
$$


holds for all $x \in[a, b]$.

Definition 1.5. Let $f \in L[a, b]$. The right-hand side and left-hand side Hadamard fractional integrals $J_{a^{+}}^{\alpha} f$ and $J_{b^{-}}^{\alpha} f$ of order $\alpha>0$ with $b>a \geq 0$ are defined by

$$
\begin{aligned}
& J_{a^{+}}^{\alpha} f(x)=\frac{1}{\Gamma(\alpha)} \int_{a}^{x}(x-t)^{\alpha-1} f(t) d t, x>a, \\
& J_{b^{-}}^{\alpha} f(x)=\frac{1}{\Gamma(\alpha)} \int_{x}^{b}(t-x)^{\alpha-1} f(t) d t, x<b
\end{aligned}
$$

respectively where $\Gamma(\alpha)$ is the Gamma function defined by $\Gamma(\alpha)=\int_{0}^{\infty} e^{-t} t^{\alpha-1}$, $J_{a^{+}}^{0} f(x)=J_{b^{-}}^{0} f(x)=f(x)$.

Theorem 1.6. [5] Let $f: I \subset \mathbb{R} \backslash\{0\} \rightarrow \mathbb{R}$ be a harmonically convex function and $a, b \in I$ with $a<b$. If $f \in L[a, b]$ then the following inequalities hold:

$$
f\left(\frac{2 a b}{a+b}\right) \leq \frac{a b}{b-a} \int_{a}^{b} \frac{f(x)}{x^{2}} d x \leq \frac{f(a)+f(b)}{2} .
$$

In [10, İşcan and Wu represented Hermite-Hadamard's inequalities for harmonically convex functions in fractional integral form as follows:

Theorem 1.7. 10 Let $f: I \subseteq \mathbb{R}^{+} \rightarrow \mathbb{R}$ be differentiable on $I^{\circ}$, and $a, b \in I$ with $a<b$ and $f \in L[a, b]$. If $f$ is harmonically-convex on $[a, b]$, then the following inequalities for fractional integrals hold:

$$
\begin{aligned}
f\left(\frac{2 a b}{a+b}\right) & \leq \frac{\Gamma(\alpha+1)}{2}\left(\frac{a b}{a+b}\right)^{\alpha}\left\{\begin{array}{c}
J_{1 / a^{-}}^{\alpha}(f \circ h)(1 / b) \\
+J_{1 / b^{+}}^{\alpha}(f \circ h)(1 / a)
\end{array}\right\} \\
& \leq \frac{f(a)+f(b)}{2} .
\end{aligned}
$$

with $\alpha>0$ and $h(x)=1 / x$.

In [12, Chan and $\mathrm{Wu}$ represented Hermite-Hadamard-Fejér inequality for harmonically convex functions as follows:

Theorem 1.8. Suppose that $f: I \subseteq \mathbb{R} \backslash\{0\} \longrightarrow \mathbb{R}$ be harmonically-convex function and $a, b \in I$, with $a<b$. If $f \in L[a, b]$ and $g:[a, b] \subseteq \mathbb{R} \backslash\{0\} \longrightarrow \mathbb{R}$ is nonnegative, integrable and harmonically symmetric with respect to $2 a b / a+$ $b$, then

$$
f\left(\frac{2 a b}{a+b}\right) \int_{a}^{b} \frac{g(x)}{x^{2}} d x \leq \int_{a}^{b} \frac{f(x) g(x)}{x^{2}} d x \leq \frac{f(a)+f(b)}{2} \int_{a}^{b} \frac{g(x)}{x^{2}} d x
$$


In [8], İşcan and Kunt represented Hermite-Hadamard-Fejér type inequality for harmonically convex functions in fractional integral forms and established following identity as follow

Theorem 1.9. Let $f:[a, b] \longrightarrow \mathbb{R}$ be harmonically convex function with $a<b$ and $f \in L[a, b]$. If $g:[a, b] \longrightarrow \mathbb{R}$ is nonnegative, integrable and harmonically symmetric with respect to $2 a b / a+b$, then the following inequalities for fractional integrals hold:

$$
\begin{gathered}
f\left(\frac{2 a b}{a+b}\right)\left[J_{1 / a^{-}}^{\alpha}(g \circ h)(1 / b)+J_{1 / b^{+}}^{\alpha}(g \circ h)(1 / a)\right] \\
\leq\left[J_{1 / a^{-}}^{\alpha}(f g \circ h)(1 / b)+J_{1 / b^{+}}^{\alpha}(f g \circ h)(1 / a)\right] \\
\leq \frac{f(a)+f(b)}{2}\left[J_{1 / a^{-}}^{\alpha}(g \circ h)(1 / b)+J_{1 / b^{+}}^{\alpha}(g \circ h)(1 / a)\right]
\end{gathered}
$$

with $\alpha>0$ and $h(x)=1 / x, x \in\left[\frac{1}{b}, \frac{1}{a}\right]$. In [2] D. Y. Hwang found out a new identity and by using this identity, established a new inequalities. Then in [14 İ. İscan and S. Turhan used this identity for harmonically convex functions and obtain generalized new inequalities. In this paper, we established a new inequality similar to inequality in [14] and then we obtained some new and general integral inequalities for differentiable harmonically-convex functions using this lemma. The following sections, let the notion, $L(t)=\frac{a H}{t H+(1-t) a}, U(t)=\frac{b H}{t H+(1-t) b}$ and $H=H(a, b)=\frac{2 a b}{a+b}$.

\section{Main result}

Throughout this section, let $\|g\|_{\infty}=\sup _{x \in[a, b]}|g(x)|$, for the continuous function $g:[a, b] \longrightarrow \mathbb{R}$ be differentiable mapping $I^{o}$, where $a, b \in I$ with $a \leq b$, and $h:[a, b] \longrightarrow[0, \infty)$ be differentiable mapping.

Lemma 2.1. Let $f: I \subseteq \mathbb{R}_{+}=(0, \infty) \longrightarrow \mathbb{R}$ be a differentiable function on $I^{o}, a, b \in I^{o}$ with $a<b$. If $h:[a, b] \longrightarrow[0, \infty)$ is a differentiable function and $f^{\prime} \in L([a, b])$, the following inequality holds:

$$
\begin{array}{r}
\left(\frac{f(a)+f(b)}{2}\right) h(a)-f(H) h(b) \\
+\frac{b-a}{4 a b}\left\{\begin{array}{c}
\int_{0}^{1}\left[h^{\prime}(L(t))(L(t))^{2}+h^{\prime}(U(t))(U(t))^{2}\right] \\
\times[f(L(t))+f(U(t))] d t
\end{array}\right\} \\
=\frac{b-a}{4 a b}\left\{\begin{array}{c}
\int_{0}^{1}[h(L(t))-h(U(t))+h(b)] \\
\times\left[-f^{\prime}(L(t))(L(t))^{2}+f^{\prime}(U(t))(U(t))^{2}\right] d t
\end{array}\right\} .
\end{array}
$$


Proof. By the integration by parts, we have

$$
\begin{aligned}
I_{1} & =\int_{0}^{1}[h(L(t))-h(U(t))+h(b)] d(f(L(t))) \\
& =\left.[h(L(t))-h(U(t))+h(b)] f(L(t))\right|_{0} ^{1} \\
& -\int_{0}^{1} f(L(t))\left[h^{\prime}(L(t)) L^{\prime}(t)-h^{\prime}(U(t)) U^{\prime}(t)\right] d t \\
& =h(a) f(a)-h(b) f(H)-\int_{0}^{1} f(L(t))\left[h^{\prime}(L(t)) L^{\prime}(t)-h^{\prime}(U(t)) U^{\prime}(t)\right] d t
\end{aligned}
$$

and

$$
\begin{aligned}
I_{2} & =\int_{0}^{1}[h(L(t))-h(U(t))+h(b)] d(f(U(t))) \\
& =\left.[h(L(t))-h(U(t))+h(b)] f(U(t))\right|_{0} ^{1} \\
& -\int_{0}^{1} f(U(t))\left[h^{\prime}(L(t)) L^{\prime}(t)-h^{\prime}(U(t)) U^{\prime}(t)\right] d t \\
& =h(a) f(b)-h(b) f(H)-\int_{0}^{1} f(U(t))\left[h^{\prime}(L(t)) L^{\prime}(t)-h^{\prime}(U(t)) U^{\prime}(t)\right] d t
\end{aligned}
$$

Therefore

$$
\begin{aligned}
\frac{I_{1}+I_{2}}{2}= & \left(\frac{f(a)+f(b)}{2}\right) h(a)-h(b) f(H) \\
& -\frac{b-a}{4 a b}\left\{\begin{array}{c}
\int_{0}^{1}\left[h^{\prime}(L(t))(L(t))^{2}+h^{\prime}(U(t))(U(t))^{2}\right] \\
\times[f(L(t))+f(U(t))] d t
\end{array}\right\}
\end{aligned}
$$

This complete the proof.

Theorem 2.2. Let $f: I \subseteq \mathbb{R}_{+}=(0, \infty) \longrightarrow \mathbb{R}$ be differentiable function on $I^{o}$ and $a, b \in I^{o}$ with $a<b$. If $h:[a, b] \longrightarrow[0, \infty)$ is a differentiable function and $\left|f^{\prime}\right|$ is harmonically convex on $[a, b]$, the following inequality holds

$$
\begin{aligned}
& \mid\left(\frac{f(a)+f(b)}{2}\right) h(a)-h(b) f\left(\frac{2 a b}{a+b}\right) \\
& +\frac{1}{2}\left[\int_{a}^{b} f(x) h^{\prime}(x) d x+\int_{a}^{b} f(x) h^{\prime}\left(\frac{H x}{2 x-H}\right)\left(\frac{H}{2 x-H}\right)^{2} d x\right] \mid
\end{aligned}
$$




$$
\leq \frac{b-a}{4 a b}\left[\zeta_{1}(a, b)\left|f^{\prime}(a)\right|+\zeta_{2}(a, b)\left|f^{\prime}(H)\right|+\zeta_{3}(a, b)\left|f^{\prime}(b)\right|\right]
$$

where

$$
\begin{aligned}
\zeta_{1}(a, b) & =\int_{0}^{1} t(L(t))^{2}|h(L(t))-h(U(t))+h(b)| d t \\
\zeta_{2}(a, b) & =\int_{0}^{1}(1-t)|h(L(t))-h(U(t))+h(b)|\left[(L(t))^{2}+(U(t))^{2}\right] d t,(10) \\
\zeta_{3}(a, b) & =\int_{0}^{1} t(U(t))^{2}|h(L(t))-h(U(t))+h(b)| d t
\end{aligned}
$$

Proof. We get the following inequality take the absolute value to (6):

$$
\begin{aligned}
& \mid\left(\frac{f(a)+f(b)}{2}\right) h(a)-h(b) f\left(\frac{2 a b}{a+b}\right) \\
& +\frac{1}{2}\left[\int_{a}^{b} f(x) h^{\prime}(x) d x+\int_{a}^{b} f(x) h^{\prime}\left(\frac{H x}{2 x-H}\right)\left(\frac{H}{2 x-H}\right)^{2} d x\right] \mid \\
& \leq \frac{b-a}{4 a b}\left\{\int_{0}^{1}|h(L(t))-h(U(t))+h(b)|\left|f^{\prime}(L(t))(L(t))^{2}\right| d t\right. \\
& \left.+\int_{0}^{1}|h(L(t))-h(U(t))+h(b)|\left|f^{\prime}(U(t))(U(t))^{2}\right| d t\right\} .
\end{aligned}
$$

Since $\left|f^{\prime}\right|$ is harmonically-convex on $[a, b]$ in $[12)$, we have for all $t \in[a, b]$ that

$$
\begin{aligned}
& \mid\left(\frac{f(a)+f(b)}{2}\right) h(a)-h(b) f\left(\frac{2 a b}{a+b}\right) \\
& +\frac{1}{2}\left[\int_{a}^{b} f(x) h^{\prime}(x) d x+\int_{a}^{b} f(x) h^{\prime}\left(\frac{H x}{2 x-H}\right)\left(\frac{H}{2 x-H}\right)^{2} d x\right] \mid \\
& \leq \frac{b-a}{4 a b}\left\{\int_{0}^{1}|h(L(t))-h(U(t))+h(b)|\left[t\left|f^{\prime}(a)\right|+(1-t)\left|f^{\prime}(H)\right|\right](L(t))^{2} d t\right. \\
& \left.+\int_{0}^{1}|h(L(t))-h(U(t))+h(b)|\left[t\left|f^{\prime}(b)\right|+(1-t)\left|f^{\prime}(H)\right|\right](U(t))^{2} d t\right\},
\end{aligned}
$$

this proof is completed. 
Corollary 2.3. Suppose that $g:[a, b] \longrightarrow[0, \infty)$ is a continuous positive mapping and geometrically symmetric with respect to $\frac{2 a b}{a+b}$ (i.e. $g(x)=g\left(\frac{1}{\frac{1}{a}+\frac{1}{b}-\frac{1}{x}}\right)$ holds for all $x \in[a, b]$ with $a<b)$. Taking

$$
h(t)=\int_{1 / t}^{1 / a}\left[\left(x-\frac{1}{b}\right)^{\alpha-1}+\left(\frac{1}{a}-x\right)^{\alpha-1}\right](g \circ \varphi)(x) d x
$$

$1 / t \in\left[\frac{1}{b}, \frac{1}{a}\right], \alpha>0$ and $\varphi(x)=\frac{1}{x}$ in Theorem 2.2, we get

$$
\begin{aligned}
& \mid\left[J_{1 / b^{+}}^{\alpha}(f g \circ \varphi)(1 / a)+J_{1 / a^{-}}^{\alpha}(f g \circ \varphi)(1 / b)\right] \\
& -f\left(\frac{2 a b}{a+b}\right)\left[J_{1 / b^{+}}^{\alpha}(g \circ \varphi)(1 / a)+J_{1 / a^{-}}^{\alpha}(g \circ \varphi)(1 / b)\right] \mid \\
& \leq \frac{(b-a)^{\alpha+1}\|g\|_{\infty}}{2(a b)^{\alpha+1} \Gamma(\alpha+1)}\left[C_{1}(\alpha)\left|f^{\prime}(a)\right|+C_{2}(\alpha)\left|f^{\prime}(H)\right|+C_{3}(\alpha)\left|f^{\prime}(b)\right|\right]
\end{aligned}
$$

where

$$
\begin{aligned}
& C_{1}(\alpha)=\int_{0}^{1}\left[1-\left(\frac{1+t}{2}\right)^{\alpha}+\left(\frac{1-t}{2}\right)^{\alpha}\right] t(L(t))^{2} d t \\
& C_{2}(\alpha)=\int_{0}^{1}\left[1-\left(\frac{1+t}{2}\right)^{\alpha}+\left(\frac{1-t}{2}\right)^{\alpha}\right](1-t)\left[(L(t))^{2}+(U(t))^{2}\right] d t \\
& C_{3}(\alpha)=\int_{0}^{1}\left[1-\left(\frac{1+t}{2}\right)^{\alpha}+\left(\frac{1-t}{2}\right)^{\alpha}\right] t(U(t))^{2} d t .
\end{aligned}
$$

Proof. If we write $h(t)=\int_{1 / t}^{1 / a}\left[\left(x-\frac{1}{b}\right)^{\alpha-1}+\left(\frac{1}{a}-x\right)^{\alpha-1}\right] g \circ \varphi(x) d x$ for all $1 / t \in$ $\left[\frac{1}{b}, \frac{1}{a}\right]$ and $\varphi(x)=1 / x$ in 13$)$, we have

$$
\begin{aligned}
& \mid \frac{1}{2}\left[\int_{a}^{b}\left[\left(\frac{1}{a}-x\right)^{\alpha-1}+\left(x-\frac{1}{b}\right)^{\alpha-1}\right] \frac{f(x) g(x)}{x^{2}} d x\right. \\
& \left.+\int_{a}^{b}\left[\left(\frac{1}{a}-x\right)^{\alpha-1}+\left(x-\frac{1}{b}\right)^{\alpha-1}\right] \frac{f(x) g\left(\frac{H x}{2 x-H}\right)}{x^{2}} d x\right] \\
& -f\left(\frac{2 a b}{a+b}\right) \int_{a}^{b}\left[\left(\frac{1}{a}-x\right)^{\alpha-1}+\left(x-\frac{1}{b}\right)^{\alpha-1}\right] \frac{g(x)}{x^{2}} d x \mid .
\end{aligned}
$$


From $g(x)$ is harmonically symmetric with respect to $x=2 a b / a+b$, we get

$$
\begin{aligned}
& \mid \int_{a}^{b}\left[\left(\frac{1}{a}-x\right)^{\alpha-1}+\left(x-\frac{1}{b}\right)^{\alpha-1}\right] \frac{f(x) g(x)}{x^{2}} d x \\
& -f\left(\frac{2 a b}{a+b}\right) \int_{a}^{b}\left[\left(\frac{1}{a}-x\right)^{\alpha-1}+\left(x-\frac{1}{b}\right)^{\alpha-1}\right] \frac{g(x)}{x^{2}} d x \mid \\
& =\left|\begin{array}{c}
\Gamma(\alpha)\left[J_{1 / b^{+}}^{\alpha}(f g \circ \varphi)(1 / a)+J_{1 / a^{-}}^{\alpha}(f g \circ \varphi)(1 / b)\right] \\
-\Gamma(\alpha)\left[J_{1 / b^{+}}^{\alpha} g \circ \varphi(1 / a)+J_{1 / a^{-}}^{\alpha} g \circ \varphi(1 / b)\right] f\left(\frac{2 a b}{a+b}\right)
\end{array}\right| .
\end{aligned}
$$

On the other hand, right side of inequality 13

$$
\left.\begin{array}{c}
\leq \frac{b-a}{4 a b}\left\{\int_{0}^{1}\left|\begin{array}{l}
\mid \int_{1 / L(t)}^{1 / a}\left[\left(\frac{1}{a}-x\right)^{\alpha-1}+\left(x-\frac{1}{b}\right)^{\alpha-1}\right](g \circ \varphi)(x) d x \\
-\int_{1 / U(t)}^{1 / a}\left[\left(\frac{1}{a}-x\right)^{\alpha-1}+\left(x-\frac{1}{b}\right)^{\alpha-1}\right](g \circ \varphi)(x) d x \\
+\int_{1 / b}^{1 / a}\left[\left(\frac{1}{a}-x\right)^{\alpha-1}+\left(x-\frac{1}{b}\right)^{\alpha-1}\right](g \circ \varphi)(x) d x
\end{array}\right| A d t\right. \\
+\int_{0}^{1}\left|\begin{array}{c}
\int_{1 / L(t)}^{1 / a} \\
-\int_{1 / U(t)}^{1 / a}\left[\left(\frac{1}{a}-x\right)^{\alpha-1}+\left(x-\frac{1}{b}\right)^{\alpha-1}\right](g \circ \varphi)(x) d x \\
+\int_{1 / b}^{1 / a}\left[\left(\frac{1}{a}-x\right)^{\alpha-1}+\left(x-\frac{1}{b}\right)^{\alpha-1}\right](g \circ \varphi)(x) d x
\end{array}\right| B d t
\end{array}\right\} .
$$

Where

$$
A=\left[t f^{\prime}(a)+(1-t) f^{\prime}(H)\right](L(t))^{2}
$$

and

$$
B=\left[t f^{\prime}(b)+(1-t) f^{\prime}(H)\right](U(t))^{2}
$$

Since $g(x)$ is symmetric to $x=2 a b / a+b$, we have

$$
\mid \begin{gathered}
\int_{1 / L(t)}^{1 / a}\left[\left(\frac{1}{a}-x\right)^{\alpha-1}+\left(x-\frac{1}{b}\right)^{\alpha-1}\right](g \circ \varphi)(x) d x \\
-\int_{1 / U(t)}^{1 / a}\left[\left(\frac{1}{a}-x\right)^{\alpha-1}+\left(x-\frac{1}{b}\right)^{\alpha-1}\right](g \circ \varphi)(x) d x \\
+\int_{1 / b}^{1 / a}\left[\left(\frac{1}{a}-x\right)^{\alpha-1}+\left(x-\frac{1}{b}\right)^{\alpha-1}\right](g \circ \varphi)(x) d x
\end{gathered}
$$




$$
\begin{aligned}
& =\int_{1 / L(t)}^{1 / a}\left[\left(\frac{1}{a}-x\right)^{\alpha-1}+\left(x-\frac{1}{b}\right)^{\alpha-1}\right](g \circ \varphi)(x) d x \\
& +\int_{1 / b}^{1 / U(t)}\left[\left(\frac{1}{a}-x\right)^{\alpha-1}+\left(x-\frac{1}{b}\right)^{\alpha-1}\right](g \circ \varphi)(x) d x
\end{aligned}
$$

and

$$
\begin{aligned}
& \int_{1 / L(t)}^{1 / a}\left[\left(\frac{1}{a}-x\right)^{\alpha-1}+\left(x-\frac{1}{b}\right)^{\alpha-1}\right](g \circ \varphi)(x) d x \\
& =\int_{1 / b}^{1 / U(t)}\left[\left(\frac{1}{a}-x\right)^{\alpha-1}+\left(x-\frac{1}{b}\right)^{\alpha-1}\right](g \circ \varphi)(x) d x
\end{aligned}
$$

for all $t \in[0,1]$ and $\varphi(x)=1 / x$. By $(15)-(18)$, we have

$$
\begin{aligned}
& \left|\left[J_{a^{+}}^{\alpha}(f g)(b)+J_{b^{-}}^{\alpha}(f g)(a)\right]-\left[J_{a^{+}}^{\alpha} g(b)+J_{b^{-}}^{\alpha} g(a)\right] f\left(\frac{2 a b}{a+b}\right)\right| \\
& \leq \frac{b-a}{2 a b \Gamma(\alpha)}\left\{\int_{0}^{1}\left|\int_{1 / L(t)}^{1 / a}\left[\left(\frac{1}{a}-x\right)^{\alpha-1}+\left(x-\frac{1}{b}\right)^{\alpha-1}\right](g \circ \varphi)(x) d x\right|\right.
\end{aligned}
$$

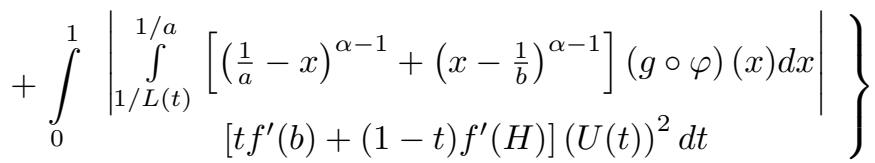

$$
\begin{aligned}
& \leq \frac{(b-a)\|g\|_{\infty}}{2 a b \Gamma(\alpha)}\left\{\int_{0}^{1}\left(\int_{1 / L(t)}^{1 / a}\left[\left(\frac{1}{a}-x\right)^{\alpha-1}+\left(x-\frac{1}{b}\right)^{\alpha-1}\right] d x\right)\right. \\
& \left.+\int_{0}^{1} \underset{\substack{1 / L(t) \\
\left[t f^{\prime}(b)+(1-t) f^{\prime}(H)\right](U(t))^{2} d t}}{\left.\left.\left(\frac{1}{a}-x\right)^{\alpha-1}+\left(x-\frac{1}{b}\right)^{\alpha-1}\right] d x\right)}\right\} .
\end{aligned}
$$

In the last inequality, we calculate

$$
\int_{1 / L(t)}^{1 / a}\left[\left(\frac{1}{a}-x\right)^{\alpha-1}+\left(x-\frac{1}{b}\right)^{\alpha-1}\right] d x=\frac{(b-a)^{\alpha}}{\alpha(a b)^{\alpha}}\left[1-\left(\frac{1+t}{2}\right)^{\alpha}+\left(\frac{1-t}{2}\right)^{\alpha}\right] .
$$

A combination of (19) and (20), we have (14). This complete is proof. 
Corollary 2.4. In Corollary 2.3, (1) If we take $\alpha=1$, we obtain following Hermite-Hadamard-Fejér Type inequality for harmonically-convex functions related to (14):

$$
\begin{aligned}
& \left|\int_{a}^{b} f(x) \frac{g(x)}{x^{2}} d x-f\left(\frac{2 a b}{a+b}\right) \int_{a}^{b} \frac{g(x)}{x^{2}} d x\right| \\
& \leq \frac{(b-a)^{2}}{2(a b)^{2}}\|g\|_{\infty}\left[C_{1}(1)\left|f^{\prime}(a)\right|+C_{2}(1)\left|f^{\prime}(H)\right|+C_{3}(1)\left|f^{\prime}(b)\right|\right]
\end{aligned}
$$

where

$$
\begin{aligned}
C_{1}(1) & =\int_{0}^{1} t(1-t)(L(t))^{2} d t \\
C_{2}(1) & =\int_{0}^{1}(1-t)^{2}\left[(L(t))^{2}+(U(t))^{2}\right] d t, \\
C_{3}(1) & =\int_{0}^{1} t(1-t)(U(t))^{2} d t .
\end{aligned}
$$

(2)If we take $g(x)=1$, we obtain following inequality is related to 14):

$$
\begin{aligned}
& \left|\frac{(a b)^{\alpha} \Gamma(\alpha+1)}{2(b-a)^{\alpha}}\left[J_{1 / b^{+}}^{\alpha}(f \circ \varphi)(1 / a)+J_{1 / a^{-}}^{\alpha}(f \circ \varphi)(1 / b)\right]-f\left(\frac{2 a b}{a+b}\right)\right| \\
& \leq \frac{(b-a)}{4 a b}\left[C_{1}(\alpha)\left|f^{\prime}(a)\right|+C_{2}(\alpha)\left|f^{\prime}(H)\right|+C_{3}(\alpha)\left|f^{\prime}(b)\right|\right] .
\end{aligned}
$$

(3) If we take $g(x)=1$ and $\alpha=1$, we obtain following inequality is related to (14):

$$
\begin{aligned}
& \left|\frac{a b}{b-a} \int_{a}^{b} \frac{f(x)}{x} d x-f\left(\frac{2 a b}{a+b}\right)\right| \\
& \leq \frac{(b-a)}{4 a b}\left[C_{1}(1)\left|f^{\prime}(a)\right|+C_{2}(1)\left|f^{\prime}(H)\right|+C_{3}(1)\left|f^{\prime}(b)\right|\right] .
\end{aligned}
$$

Theorem 2.5. Let $f: I \subseteq \mathbb{R}_{+}=(0, \infty) \longrightarrow \mathbb{R}$ be a differentiable function on $I^{o}, a, b \in I^{o}$ with $a<b$. If $h:[a, b] \longrightarrow[0, \infty)$ is a differentiable function and $\left|f^{\prime}\right|^{q}$ is harmonically convex on $[a, b]$ for $q \geq 1$, the following inequality holds

$$
\begin{aligned}
& \mid\left(\frac{f(a)+f(b)}{2}\right) h(a)-h(b) f\left(\frac{2 a b}{a+b}\right) \\
& +\frac{1}{2}\left[\int_{a}^{b} f(x) h^{\prime}(x) d x+\int_{a}^{b} f(x) h^{\prime}\left(\frac{H x}{2 x-H}\right)\left(\frac{H}{2 x-H}\right)^{2} d x\right] \mid
\end{aligned}
$$




$$
\begin{aligned}
& \leq \frac{(b-a)}{4 a b}\left\{\begin{array}{c}
\left(\int_{0}^{1}|h(L(t))-h(U(t))+h(b)| d t\right)^{1-\frac{1}{q}} \times \\
\left(\int_{0}^{1} \times\left(t(L(t))^{2 q}\left|f^{\prime}(a)\right|^{q}+(1-t)(L(t))^{2 q}\left|f^{\prime}(H)\right|^{q}\right) d t\right)^{\frac{1}{q}}
\end{array}\right. \\
& +\left(\int_{0}^{1}|h(L(t))-h(U(t))+h(b)| d t\right)^{1-\frac{1}{q}} \times \\
& \left.\left(\int_{0}^{1} \times\left(t(U(t))^{2 q}\left|f^{\prime}(b)\right|^{q}+(1-t)(U(t))^{2 q}\left|f^{\prime}(H)\right|^{q}\right) d t\right)^{\frac{1}{q}}\right\}
\end{aligned}
$$

Proof. Continuing from $(12)$ in proof of Theorem 2.2 , the power mean inequality and using the fact that $\left|f^{\prime}\right|^{d}$ is harmonically convex on $[a, b]$, we get the required result. This completes the proof of the theorem.

Corollary 2.6. Let $g:[a, b] \longrightarrow[0, \infty)$ be a positive continuous mapping and harmonically symmetric with respect to $2 a b / a+b$ (i.e. $g\left(\frac{1}{\frac{1}{a}+\frac{1}{b}-\frac{1}{x}}\right)=g(x)$ holds for all $x \in[a, b]$ with $a<b$ ). If we take

$$
h(t)=\int_{1 / t}^{1 / a}\left[\left(x-\frac{1}{b}\right)^{\alpha-1}+\left(\frac{1}{a}-x\right)^{\alpha-1}\right](g \circ \varphi)(x) d x
$$

$, t \in[a, b], \varphi(x)=\frac{1}{x}$ in Theorem 6, we obtain

$$
\begin{aligned}
& \mid\left[J_{1 / b^{+}}^{\alpha}(f g \circ \varphi)(1 / a)+J_{1 / a^{-}}^{\alpha}(f g \circ \varphi)(1 / b)\right] \\
& -f\left(\frac{2 a b}{a+b}\right)\left[J_{1 / b^{+}}^{\alpha}(g \circ \varphi)(1 / a)+J_{1 / a^{-}}^{\alpha}(g \circ \varphi)(1 / b)\right] \mid \\
& \leq \frac{(b-a)^{\alpha+1}\|g\|_{\infty}}{2(a b)^{\alpha+1} \Gamma(\alpha+1)}\left[2-\frac{4}{\alpha+1}+\frac{2^{2-\alpha}}{\alpha+1}\right]^{1-\frac{1}{q}} \\
& {\left[C_{1}(\alpha, q)\left|f^{\prime}(a)\right|^{q}+C_{2}(\alpha, q)\left|f^{\prime}(H)\right|^{q}+C_{3}(\alpha, q)\left|f^{\prime}(b)\right|^{q}\right]^{\frac{1}{q}}}
\end{aligned}
$$

where for $q \geq 1$

$$
\begin{aligned}
& C_{1}(\alpha, q)=\int_{0}^{1}\left[1-\left(\frac{1+t}{2}\right)^{\alpha}+\left(\frac{1-t}{2}\right)^{\alpha}\right] t(L(t))^{2 q} d t \\
& C_{2}(\alpha, q)=\int_{0}^{1}\left[1-\left(\frac{1+t}{2}\right)^{\alpha}+\left(\frac{1-t}{2}\right)^{\alpha}\right](1-t)\left((L(t))^{2 q}+(U(t))^{2 q}\right) d t \\
& C_{3}(\alpha, q)=\int_{0}^{1}\left[1-\left(\frac{1+t}{2}\right)^{\alpha}+\left(\frac{1-t}{2}\right)^{\alpha}\right] t(U(t))^{2 q} d t
\end{aligned}
$$


Proof. We use the equality (20) of Corollary 1 and (24) in Theorem 2.5.

$$
\begin{aligned}
& \mid\left[J_{1 / b^{+}}^{\alpha}(f g \circ \varphi)(1 / a)+J_{1 / a^{-}}^{\alpha}(f g \circ \varphi)(1 / b)\right] \\
& -f\left(\frac{2 a b}{a+b}\right)\left[J_{1 / b^{+}}^{\alpha}(g \circ \varphi)(1 / a)+J_{1 / a^{-}}^{\alpha}(g \circ \varphi)(1 / b)\right] \mid \\
& \leq \frac{(b-a)^{\alpha+1}\|g\|_{\infty}}{2(a b)^{\alpha+1} \Gamma(\alpha+1)} \times \\
& \left\{\begin{array}{c}
\left(\int_{0}^{1}\left[1-\left(\frac{1+t}{2}\right)^{\alpha}+\left(\frac{1-t}{2}\right)^{\alpha}\right] d t\right)^{1-\frac{1}{q}} \times \\
{\left[1-\left(\frac{1+t}{2}\right)^{\alpha}+\left(\frac{1-t}{2}\right)^{\alpha}\right]} \\
\left(\int_{0}^{1}\left(t(L(t))^{2 q}\left|f^{\prime}(a)\right|^{q}+(1-t)(L(t))^{2 q}\left|f^{\prime}(H)\right|^{q}\right) d t\right.
\end{array}\right)^{\frac{1}{q}} \\
& \left(\int_{0}^{1}\left[1-\left(\frac{1+t}{2}\right)^{\alpha}+\left(\frac{1-t}{2}\right)^{\alpha}\right] d t\right)^{1-\frac{1}{q}} \times \\
& \left.+\left(\int_{0}^{1}\left(t(U(t))^{2 q}\left|f^{\prime}(b)\right|^{q}+(1-t)(U(t))^{2 q}\left|f^{\prime}(H)\right|^{q}\right) d t\right)^{\frac{1}{q}}\right\} \\
& \leq \frac{(b-a)^{\alpha+1}\|g\|_{\infty}}{2(a b)^{\alpha+1} \Gamma(\alpha+1)}\left[1-\frac{2}{\alpha+1}+\frac{2^{1-\alpha}}{\alpha+1}\right]^{1-\frac{1}{q}} \times \\
& {\left[\begin{array}{c}
\left(1-\left(\frac{1+t}{2}\right)^{\alpha}+\left(\frac{1-t}{2}\right)^{\alpha}\right] \times \\
\left.\int_{0}^{1}\left[t(L(t))^{2 q}\left|f^{\prime}(a)\right|^{q}+(1-t)(L(t))^{2 q}\left|f^{\prime}(H)\right|^{q}\right] d t\right)^{\frac{1}{q}} \\
+\left(\int_{0}^{1}\left[t(U(t))^{2 q}\left|f^{\prime}(b)\right|^{q}+(1-t)(U(t))^{2 q}\left|f^{\prime}(H)\right|^{q}\right] d t\right)^{\frac{1}{q}}
\end{array}\right]}
\end{aligned}
$$

By the power-mean inequality $\left(a^{r}+b^{r}<2^{1-r}(a+b)^{r}\right.$ for $\left.a>0, b>0, \quad r \leq 1\right)$, we have

$$
\begin{aligned}
& \leq \frac{(b-a)^{\alpha+1}\|g\|_{\infty}}{2(a b)^{\alpha+1} \Gamma(\alpha+1)}\left[2-\frac{4}{\alpha+1}+\frac{2^{2-\alpha}}{\alpha+1}\right]^{1-\frac{1}{q}} \\
& \times\left[\int_{0}^{1}\left(\begin{array}{c}
{\left[1-\left(\frac{1+t}{2}\right)^{\alpha}+\left(\frac{1-t}{2}\right)^{\alpha}\right] t(L(t))^{2 q}\left|f^{\prime}(a)\right|^{q}} \\
+\left[1-\left(\frac{1+t}{2}\right)^{\alpha}+\left(\frac{1-t}{2}\right)^{\alpha}\right](1-t)\left(\begin{array}{c}
(L(t))^{2 q} \\
+(U(t))^{2 q}
\end{array}\right)\left|f^{\prime}(H)\right|^{q} \\
+\left[1-\left(\frac{1+t}{2}\right)^{\alpha}+\left(\frac{1-t}{2}\right)^{\alpha}\right] t(U(t))^{2 q}\left|f^{\prime}(b)\right|^{q}
\end{array}\right) d t\right]^{\frac{1}{q}} .
\end{aligned}
$$

Corollary 2.7. When $\alpha=1, g(x)=1$ is taken in Corollary 2.6, we obtain:

$$
\left|\frac{a b}{b-a} \int_{a}^{b} \frac{f(x)}{x^{2}} d x-f\left(\frac{2 a b}{a+b}\right)\right|
$$




$$
\leq \frac{(b-a)}{4 a b}\left[C_{1}(1, q)\left|f^{\prime}(a)\right|^{q}+C_{2}(1, q)\left|f^{\prime}(H)\right|^{q}+C_{3}(1, q)\left|f^{\prime}(b)\right|^{q}\right]^{\frac{1}{q}} .
$$

This proof is completed.

\section{Competing Interests}

The author(s) do not have any competing interests in the manuscript.

\section{REFERENCES}

1. Hwang, D. Y. (2011). Some inequalities for differentiable convex mapping with application to weighted trapezoidal formula and higher moments of random variables. Applied Mathematics and Computation, 217(23), 9598-9605. https://doi.org/10.1016/j.amc.2011.04.036

2. Hwang, D. Y. (2014). Some inequalities for differentiable convex mapping with application to weighted midpoint formula and higher moments of random variables. Applied Mathematics and Computation, 232, 68-75. https://doi.org/10.1016/j.amc.2014.01.050

3. Dragomir, S. S. (2012). HermiteHadamards type inequalities for convex functions of selfadjoint operators in Hilbert spaces. Linear Algebra and Its Applications, 436(5), 1503-1515. https://doi.org/10.1016/j.laa.2011.08.050

4. Dragomir, S. S., \& Pearce, C. E. M. Selected topics on Hermite-Hadamard type inequalities and applications, RGMIA Monographs, 2000. Available on line at: http://rgmia. vu. edu. au/monographs/hermite hadamard. html.

5. Chen, F., \& Wu, S. (2014). Some Hermite-Hadamard type inequalities for harmonically s-convex functions. The Scientific World Journal, 2014. doi: 10.1155/2014/279158

6. İşcan İ. (2014). Hermite-Hadamard type inequaities for harmonically functions. Hacettepe Journal of Mathematics and Statistic, 43 (6), 935-942. https://doi.org/10.1016/j.amc.2014.04.020

7. İ. İşcan, M. Kunt. (2015). Fejér and Hermite-Hadamard-Fejér type inequalities for harmonically s-convex functions via Fractional Integrals, The Australian Journal of Mathematical Analysis and Applcations, Vol: 12(1), Article 10, pp 1-6. http://dx.doi.org/10.20852/ntmsci.2016320378

8. İ. İşcan, M. Kunt. (2015) . Hermite-Hadamard-Fejér type inequalities for harmonically convex functions via fractional integrals, RGMIA Research Report Collection, 18, Article 107, pp.1-16.

9. İ. İşcan, Ostrowski type inequalities for harmonically s-convex functions, Konuralp Journal Mathematics, Volume 3, No. 1 (2015), pp. 63-74.

10. İ. İşcan, S. Wu, Hermite-Hadamard type inequalities for harmonically-convex functions via fractional integrals, Applied Mathematics and Computation, 238 (2014), 237-244.

11. F. Chen, Extensions of the Hermite Hadamard inequality for harmonically convex functions viia fractional integrals, Applied Mathematics and Computation, 268 (2015), 121-128.

12. F. Chen and S. Wu, Fejér and Hermite-Hadamard-Fejér type inequalities for harmonically convex functions, Journal of Applied Mathematics, Volume 2014, Article ID 386806.

13. Latif, M. A., Dragomir, S. S., \& Momoniat, E. (2012). Fejr type inequalities for harmonically-convex functions with applications. Journal of Applied Analysis and Computation (Accepted).

14. İşcan, I., Turhan, S., \& Maden, S. (2015). Some Hermite-Hadamard-Fejer type inequalities for harmonically convex functions via fractional integral. arXiv preprint arXiv:1511.06617.

15. Niculescu, C. P. (2003). Convexity according to means. Mathematical Inequalities and Applications, 6, 571-580. http://dx.doi.org/10.7153/mia-06-53

Sercan Turhan

Dereli Vocational High School, Giresun University, 28100, Giresun, Turkey. 
e-mail: sercanturhan28@gmail.com

İmdat İşcan

Department of Mathematics, Faculty of Arts and Sciences, Giresun University, 28100, Giresun, Turkey.

e-mail: imdat.sercan.turhan@giresun.edu.tr 